\title{
Déterminants de l'adoption des techniques de lutte contre l'érosion hydrique en zone cotonnière du Cameroun
}

\author{
Y. Ngondjeb ${ }^{1 *}$ P. Nje ${ }^{2}$ M. Havard ${ }^{3}$
}

\author{
Mots-clés \\ Adoption de l'innovation - \\ Aménagement de bassin versant - \\ Modèle économétrique - Lutte \\ antiérosion - Cameroun.
}

\begin{abstract}
Résumé
Dans la zone cotonnière du Cameroun, la pression foncière croissante a conduit à une augmentation des superficies cultivées et accentué l'érosion des sols causée par l'intensité des pluies sur des sols mal protégés. Depuis deux décennies, les aménagements antiérosifs ont été diffusés, mais peu d'études ont analysé les facteurs favorisant leur adoption. L'objectif de cette étude a été $d^{\prime}$ 'identifier les facteurs qui ont influé sur l'adoption et l'intensité d'adoption des aménagements antiérosifs par les agriculteurs. Une enquête a été effectuée dans le cadre du projet Eau-Sol-Arbre (ESA) entre 2008 et 2009 auprès de 303 agriculteurs de la zone cotonnière. Les données de cette enquête ont été analysées à l'aide d'un modèle Tobit II. Les résultats ont montré que, parmi les variables étudiées, celles qui ont influencé la décision d'adoption des aménagements antiérosifs par les agriculteurs ont été (a) I'âge des agriculteurs, (b) la perception des problèmes d'érosion par l'agriculteur, (c) leur appartenance à un groupe (religion, ethnie), (d) la disponibilité en main d'œuvre et (e) la tenure foncière. Les facteurs expliquant l'intensité de cette adoption par les agriculteurs ont été le nombre d'années écoulées depuis leur adoption des aménagements et leur mode d'accès au foncier. Le niveau d'instruction n'a influé ni sur l'adoption ni sur l'intensité de cette adoption. Afin de mieux orienter les actions futures, les programmes de lutte contre l'érosion doivent tenir compte de ces résultats dans leurs méthodes d'intervention, mais aussi dans les évaluations d'impacts socio-économiques.
\end{abstract}

\section{INTRODUCTION}

En zone cotonnière soudano-sahélienne du Cameroun, l'agriculture est perçue comme un facteur majeur de dégradation de l'environnement et plus particulièrement comme favorisant l'érosion des sols $(1,11)$. Dans cette zone, les sols ferrugineux tropicaux ont une fertilité chimique et physique intrinsèque limitée. Leur teneur réduite en argile en surface, leur faible taux de matière organique et d'azote, leur capacité de rétention d'eau limitée et leur $\mathrm{pH}$

\footnotetext{
1. Centre de recherche en économie et gestion, faculté des Sciences économiques et de Gestion, Université de Yaoundé II, Cameroun.

2. Université du Québec en Outaouais, Gatineau, Canada.

3. Cirad, UMR Innovation, Montpellier, France.

* Auteur pour la correspondance

Tél. : +2376134212361; 6132769195 ; fax : +237613 8412933

E-mail : njedora@gmail.com
}

légèrement acide les rendent sensibles à l'érosion (5). Cette érosion s'accentue avec l'augmentation rapide de la population agricole due à la croissance démographique et aux migrations (30), liées à la baisse de fertilité des terres dans les zones d'origine, le chômage, ou tout simplement le regroupement familial (5). Il en résulte une extension des surfaces cultivées et une augmentation du déboisement qui entrainent la réduction des périodes de jachère, une baisse de la fertilité des sols, et l'exposition accrue des sols aux pluies et aux vents. Cette érosion est encore accentuée par des techniques culturales basées sur des labours et des sarclobuttages.

Dans la zone soudano-sahélienne, depuis près de vingt ans, une série de projets font la promotion de l'agriculture de conservation avec l'appui des bailleurs de fonds en vue d'améliorer durablement la production agricole des exploitations agricoles vulnérables. Il s'agit particulièrement du projet Développement paysanal et gestion des terroirs (DPGT) entre 1994 et 2002, puis du projet EauSol-Arbre (ESA) depuis 2002. Ces projets font la promotion de 
techniques agricoles permettant de réduire les phénomènes d'érosion accélérée, de restaurer ou d'améliorer le statut organique des sols en vue de restaurer les paysages naturels, la fertilité des sols et, par conséquent, d'augmenter la production agricole (47).

Mais si l'agriculture de conservation apparaît comme une alternative intéressante pour améliorer durablement la sécurité alimentaire des ménages les plus pauvres dans les pays d'Afrique subsaharienne et d'Asie et, de ce fait, atteindre les Objectifs du Millénaire des Nations unies sur la sécurité alimentaire $(19,32)$, pour certains auteurs (16) l'effort de transfert de technologie en Afrique subsaharienne est insuffisant. Il est limité à des essais de démonstration dans les champs et, malgré des années d'investissement dans la recherche et le développement, les agriculteurs n'adoptent pas les techniques de conservation agricole $(20,26,42$, 50). Cette situation contraste avec celles des pays d'Amérique du Sud et de certains pays d'Asie $(9,20,49)$. Il convient alors pour la recherche de mieux analyser les cas d'adoption de technologies de conservation par les paysans pauvres d'Afrique subsaharienne pour en tirer des enseignements généralisables et améliorer leur taux d'adoption (33).

En fait, peu d'études ont été entreprises pour évaluer les facteurs clés qui influencent l'adoption des nouvelles techniques de gestion des sols et de l'eau par les petits exploitants agricoles (41). Il y a deux étapes essentielles dans la décision de mise en œuvre des techniques de conservation des sols par un agriculteur $(8,43)$ : la première est la décision d'adopter ou de ne pas adopter la technologie, et la seconde consiste à déterminer le niveau ou l'intensité d'utilisation de la technologie. Dans la zone soudano-sahélienne du Cameroun, les agriculteurs chez qui les techniques de conservation ont été introduites ont été sélectionnés par des programmes de vulgarisation du fait de leur vulnérabilité à leur environnement de production. Il ont ensuite bénéficié d'intrants agricoles et d'appuiconseil approprié pour les inciter à adopter ces techniques (30). Pourtant certains d'entre eux ont refusé de les adopter alors que d'autres ont adopté spontanément les techniques en s'inspirant de ce que faisaient leurs voisins. Parmi les agriculteurs qui ont adopté ces techniques, nombreux sont ceux qui les ont adaptées à leurs conditions de production. Des évaluations récentes suggèrent une appropriation progressive des différents composants des techniques de conservation des sols (1).

Cependant, il est nécessaire de mieux comprendre pourquoi certains agriculteurs adoptent ces techniques et d'autres pas. Il est essentiel de comprendre à la fois les caractéristiques de l'agriculteur et des exploitations qui sont susceptibles d'affecter le niveau d'adoption de ces techniques. La présente étude visait donc à identifier les déterminants de l'adoption des techniques de lutte contre l'érosion et l'intensité de cette adoption par les agriculteurs depuis leur vulgarisation par les projets de développement, afin de mieux orienter les actions futures de lutte contre l'érosion des sols en Afrique.

\section{MATERIEL ET METHODES}

Sur les 117 villages de la zone d'intervention du projet ESA dans le bassin versant du lac Lagdo, dix ont été sélectionnés pour l'analyse des facteurs d'adoption. Les critères pris en compte pour cette sélection ont été l'ancienneté des aménagements (au moins trois ans), la taille de la population (au moins 300 habitants), le degré élevé d'activité agricole et cotonnière, la vulnérabilité des sols à l'érosion, et l'accessibilité des villages. Ces aménagements antiérosifs sont aussi une des solutions proposées pour réduire l'ensablement qui affecte le lac Lagdo (36).

\section{Choix de l'échantillonnage et collecte des données}

Le tirage systématique utilisé pour sélectionner les agriculteurs chefs d'exploitation (CE) s'est appuyé sur la liste des CE (base de sondage) fournie par les différents groupements de producteurs (GP) des villages sélectionnés, sachant que la majorité des CE étaient membres de ces GP. Un pas de tirage a été calculé $(5,5)$ en divisant le nombre total de CE (1 650) des GP sélectionnés par le nombre de CE à enquêter (303). Les données collectées se rapportent à la saison agricole 2008-09 et concernent les caractéristiques sociologiques, économiques et institutionnelles des ménages des CE enquêtés. La technique d'enquête a combiné aussi bien la méthode quantitative (questionnaire) que la méthode qualitative (entretiens informels et semi-structurés). Les 303 questionnaires ont été administrés par les agents techniques de terrain du projet ESA qui parlaient les langues de leur village respectif. Les interviews (questions très ouvertes) ont été réalisées auprès d'une dizaine de CE.

Les aménagements étudiés dans cet article (ados en terre, cordons pierreux et bandes antiérosives) ont été réalisés selon les courbes de niveau et donc perpendiculairement à la pente. Ils sont adaptés à différents contextes (travail à l'échelle de la parcelle individuelle ou des blocs de culture). Ces solutions antiérosives étaient complétées par la gestion des eaux en amont des zones mises en valeur, et sur les rigoles et ravines créées par l'érosion concentrée (1). Les cordons pierreux étaient des alignements de blocs de cuirasse ou de roche. Les bandes enherbées d'Andropogon gayanus (parfois remplacée par Euphorbia balsamifera) étaient du même type que la technique précédente, les blocs rocheux ayant été remplacés par des plantes vivaces. Les associations cordons pierreux ou diguettes en terre et Andropogon gayanus qui, chaque année, était fauché ou brûlé, étaient aussi pratiquées. Des études $(5,14,29)$ ont montré l'efficacité de ces techniques pour réduire l'érosion des sols. Les cordons pierreux et les ados présentent l'avantage d'avoir une bonne efficacité, même à l'échelle de la parcelle individuelle, contrairement aux aménagements en bandes antiérosives ; ces dernières sont souvent perçues par les agriculteurs comme une perte d'espace pour quelques lignes de semis (1).

\section{Modèle empirique}

Selon la théorie néoclassique, les agriculteurs adoptent de nouvelles technologies si elles leur apportent des avantages économiques nets $(44,23)$. Toutefois, il est établi que la raison pour laquelle les agriculteurs adoptent une nouvelle technologie va au-delà de la théorie néoclassique. De nombreuses études ont analysé les variables qui influencent l'adoption de nouvelles technologies dans le secteur agricole $(10,13,23,31)$. En règle générale, les variables affectant l'adoption d'une nouvelle technologie ont été classées dans les groupes suivants : a) le capital humain ou variable sociopersonnelle, b) les facteurs structurels et c) le capital social $(23,27,28)$. En ce qui concerne le capital humain, il est d'usage d'évaluer l'effet de l'âge, du sexe, de l'éducation, de l'alphabétisation, et de l'expérience et de la formation agricoles. Parmi les facteurs structurels, la taille des exploitations et le revenu des ménages ont été largement analysés. Enfin, des études récentes ont mis l'accent sur les effets de l'accès aux réseaux sociaux sur la perception d'une nouvelle technologie par l'agriculteur et leurs conséquences sur le processus d'adoption $(23,51)$.

Dans la littérature économétrique, trois modèles ont été fréquemment utilisés pour analyser l'adoption des nouvelles technologies : (a) les modèles avec probabilité linéaire, (b) la fonction logistique (Logit) et (c) les fonctions avec densité normale (probit) $(4,32)$. 
Ces modèles utilisent des variables à choix binaire comme variable dépendante. Cependant, l'utilisation d'une variable de choix binaire comme une variable dépendante ne permet pas de capturer l'intensité d'adoption de la technologie qui peut être variable entre adoptants $(6,8)$. Pour surmonter ce problème, l'utilisation du modèle Tobit II (48) a été proposée pour l'analyse de l'intensité d'adoption où la variable dépendante est continue avec une limite zéro.

L'analyse économétrique établit l'influence avérée de certaines variables explicatives de l'adoption des aménagements et de l'intensité d'adoption (représentée par la surface aménagée divisée par la surface totale). Elle apporte des informations supplémentaires susceptibles d'aider à une meilleure compréhension du processus d'adoption des aménagements par les agriculteurs, sur la base des variables décrites dans l'analyse descriptive. La recherche de ces variables explicatives considère que la probabilité d'accepter les aménagements antiérosifs est fonction des facteurs structurels, des facteurs liés au capital humain ou variables sociopersonnelles, et des facteurs sociaux ou institutionnels. Le modèle Tobit II est un modèle de régression à variable dépendante limitée, dans lequel la variable intensité d'adoption est censurée, c'est-à-dire qu'elle n'est pas observée pour les agriculteurs qui n'adoptent pas les aménagements antiérosifs, et observée, c'est-à-dire non censurée pour ceux qui adoptent les aménagements. Le modèle Tobit (ou modèle de régression normale censurée) permet de prendre en compte la censure des données concernant l'intensité d'adoption en supposant qu'à la fois les déterminants et les effets des déterminants sont identiques pour la probabilité d'adoption des techniques de lutte contre l'érosion et pour l'intensité de cette adoption $(35,48)$. Nous avons également cherché à vérifier dans cette étude si les déterminants de l'adoption des aménagements étaient identiques à ceux de l'intensité.

Les résultats obtenus ont été évalués selon les normes statistiques (significativité des paramètres estimés, coefficient de détermination, normalité des erreurs et absence de colinéarité) et les observations empiriques en relation avec les hypothèses formulées (signes des variables explicatives).

Dans un modèle, si l'on considère un vecteur d'observation Y dont on cherche à expliquer la variabilité par un vecteur de variables $\mathrm{X}$, le modèle linéaire s'écrit :

$\mathrm{Y}=\mathrm{X} \beta+\xi$

où $\beta$ est un vecteur de coefficient à estimer et $\xi$ un vecteur d'inconnu qui représente l'effet des variables inobservées. L'exogénéité est une condition nécessaire pour estimer l'équation avec les moindres carrés ordinaires. L'hypothèse d'exogénéité des instruments suppose que pour tout individu $\mathrm{i}$, lorsque ses $\mathrm{x}_{\mathrm{i}}$ changent, son terme d'erreur $\xi_{\mathrm{i}}$ n'a pas de raison de changer (ni celui des autres individus). C'est une condition très forte, dont dépendent la validité du modèle et la qualité des estimations. Cette hypothèse a deux conséquences immédiates : l'espérance des résidus est nulle et les distributions des variables des inobservées sont non corrélées. Une variable $\mathrm{x}_{\mathrm{k}}$ qui est corrélée avec les résidus $\xi$ est dite endogène.

La décision d'adoption des nouvelles technologies par les agriculteurs est supposée basée sur la maximisation de leur utilité. Soit $\mathrm{j}=1$ la nouvelle technologie et $\mathrm{j}=2$ l'ancienne technologie, $U\left(\mathrm{M}_{\mathrm{ji}}\right.$, $\mathrm{A}_{\mathrm{ji}}$ ) la fonction d'utilité du $\mathrm{i}^{\mathrm{e}}$ agriculteur. Ceci implique que l'utilité dérivable de la technologie de conservation des sols dépend de $\mathrm{M}$ qui est le vecteur des caractéristiques sociopersonnelles, structurelles et institutionnelles de l'adoptant et A le vecteur des caractéristiques associées à la technologie. Bien que la fonction d'utilité ne soit pas observable, la relation entre l'utilité dérivée de la je technologie est supposée être une fonction de vecteur observable des caractéristiques spécifiques des champs, de l'agriculteur et des technologies (par exemple, la demande de travail, la production) et le terme aléatoire qui a une moyenne de zéro.

$\mathrm{U}_{\mathrm{ji}}=\mathrm{x}_{\mathrm{j}} \mathrm{F}_{\mathrm{j}}\left(\mathrm{M}_{\mathrm{i}}, \mathrm{A}_{\mathrm{i}}\right)+\mathrm{e}_{\mathrm{ji}} \mathrm{j}=1,2 ; \mathrm{I}=1,2 \ldots \mathrm{n}$

(équation 1)

Comme les fonctions d'utilité $\mathrm{U}_{\mathrm{ji}}$ sont aléatoires, le $\mathrm{i}^{\mathrm{e}}$ agriculteur choisira l'alternative $\mathrm{j}=1$, si $\mathrm{U}_{1 \mathrm{i}}^{\mathrm{ji}}>\mathrm{U}_{2 \mathrm{i}}$ ou si la variable latente (c'est-à-dire une variable inobservée mais qui détermine complètement la réalisation de la variable indicatrice étudiée) est $\mathrm{I}=\mathrm{U}_{1 \mathrm{i}}-\mathrm{U}_{2 \mathrm{i}}>0$. La probabilité que $\mathrm{Y}$ soit égal à 1 (c'est-à-dire que l'agriculteur adopte la nouvelle technologie) est fonction des variables indépendantes :

$$
\begin{aligned}
& P_{i}=\operatorname{Pr}\left(Y_{i}=1\right)=\operatorname{Pr}\left(U_{1 i}>U_{2 i}\right) \\
& =\operatorname{Pr}\left[\left(x_{1} F_{i}\left(M_{i}, A_{i}\right)+e_{1 i}>x_{2} F_{i}\left(M_{i}, A_{i}\right)+e_{2 i}\right]\right. \\
& =\operatorname{Pr}\left[e_{1 i}-e_{2 i}>F_{i}\left(M_{i}, A_{i}\right)\left(x_{2}-x_{1}\right)\right] \\
& =\operatorname{Pr}\left[\mu_{i}>-F_{i}\left(M_{i}, A_{i}\right) \beta\right] \\
& P_{i}=F_{i}\left(X_{i}, \beta\right)
\end{aligned}
$$

où $\mathrm{X}$ est une matrice de $\mathrm{n} x \mathrm{k}$ variables explicatives $\mathrm{b}, \beta$ est un vecteur de $\mathrm{k} \mathrm{x} 1$ paramètres à estimer, $\operatorname{Pr}$ (.) est la fonction de probabilité, $\mu_{\mathrm{i}}$ est évalué à $\mathrm{X}_{\mathrm{i}}, \beta$.

La probabilité que l'agriculteur adopte la technologie est donc fonction du vecteur de variables explicatives, de paramètres inconnus et du terme d'erreur tel que décrit par l'équation 2.

La probabilité d'adoption est estimée par un modèle probit où $\mu_{\mathrm{i}}$ est un terme d'erreur normalement distribué avec une moyenne égale à zéro et un écart-type $S^{2}$ constant.

$$
\begin{aligned}
& Y_{i}=X_{i}, \beta \text { si } \mathrm{I}=X_{i} \beta+\mu_{i}>T \text { (adoption) } \\
& =0 \text { si } \mathrm{I}=X_{i} \beta+\mu_{i}<T \text { (non adoption) }
\end{aligned}
$$

où $\mathrm{Y}_{\mathrm{i}}$ est la probabilité d'adoption de la technologie de conservation des sols, I la variable latente non observée qui reflète les effets combinés des facteurs influençant l'adoption de la technologie et $\mathrm{T}$ le niveau seuil non observé.

Basées sur les procédures d'analyse multivariée du probit, les équations suivantes examinent les facteurs qui influencent l'adoption des techniques de lutte contre l'érosion :

$$
I=\beta_{0}+\beta_{1} X_{1}+\ldots \ldots \ldots \ldots \ldots \ldots \beta_{n} X_{n}
$$$$
\text { et } \mathrm{Y}=\mathrm{g}(\mathrm{I})
$$

où $\mathrm{Y}=1$ si $\mathrm{I} \geq \mathrm{T}$

$$
\mathrm{Y}=0 \text { si } \mathrm{I} \leq \mathrm{T}
$$

La procédure d'estimation en deux étapes d'Heckman (18, 22, 40) est utilisée afin de contrôler le problème de biais de sélection de l'échantillon qui se pose souvent du fait de la classification arbitraire des adoptants et des non-adoptants. En premier lieu, il est nécessaire d'estimer la probabilité d'adoption avec un modèle probit. La seconde étape consiste à réaliser l'estimation de l'intensité d'adoption. Cette estimation est obtenue par les moindres carrés ordinaires à l'aide des observations pour lesquelles $Y_{i}>0$. La procédure d'estimation d'Heckman permet ici non seulement de lever l'hypothèse d'égalité des coefficients entre la probabilité d'adoption et l'intensité, mais également de contrôler l'existence d'un biais de sélection dans la population. Le biais de sélection est lié au fait que les individus s'autosélectionnent par leur décision d'adoption des techniques de conservation. Dans ce cas, il doit exister un biais d'omission de variable dans l'équation de niveau ou d'intensité d'adoption (14) dont l'ampleur est donnée par 
l'inverse du ratio de Mills. L'introduction de cette variable dans l'équation de niveau permet de corriger le biais. Les variables caneres utilisées dans cette étude sont présentées dans le tableau I.

\section{Analyse des données}

Le logiciel CSPRO 3.3 a été utilisé pour indiquer les valeurs limites, les éventualités possibles pour chaque questionnaire et contribuer ainsi à limiter la présence de valeurs aberrantes. Le logiciel SPSS 14 a permis de produire les statistiques descriptives et STATA.9.0 le calcul de la régression. Le pourcentage d'adoptants exprime le rapport du nombre de CE ayant adopté une technique d'aménagement sur le nombre de $\mathrm{CE}$ enquêtés, rapport multiplié par 100.

\section{Variables}

\section{Définitions}

RESULTATS

\section{Caractéristiques des chefs d'exploitation ayant adopté les aménagements}

Les tableaux II à IV décrivent les variables explicatives de l'adoption.

\section{Caractéristiques sociopersonnelles}

Les caractéristiques sociopersonnelles des CE ont été appréhendées à travers leur âge, leur ethnie d'appartenance, leur religion, leur situation matrimoniale, leur perception des problèmes d'érosion et leur niveau d'éducation (tableau II). L'âge moyen des CE

\section{Tableau I}

Variables candidates pour I'explication de l'adoption et de l'intensité d'adoption des aménagements antiérosifs

Variables dépendantes
Adoption
L'agriculteur adopte la technique (1) ; il ne l'adopte pas (0)
Intensité
Intensité ou proportion d'adoption (surface aménagée divisée par surface totale)

\section{Variables explicatives}

Variables sociopersonnelles (agriculteur)

Age

Age $^{2}$

Education

Ethnie

Religion

Situation matrimoniale

Perception
L'âge en années du chef d'exploitation

Age au carré

Niveau d'éducation du chef de ménage ; (dummy ordonnées) sans niveau (0), primaire (1), secondaire et plus (2)

Mesure l'influence du groupe ethnique sur les comportements d'adoption des agriculteurs ; Toupouri (1), Moundang (2), Massa (3), Guiziga (4), Mafa (5), Guidar (6), Daba-Kola (7), autre (8)

Influence de l'appartenance à un groupe religieux ; chrétien (1), musulman (2), animiste (3)

Célibataire (1), marié monogame (2), marié polygame (3), autre (4)

Erosion des sols ; I'agriculteur perçoit les problèmes d'érosion sur ses parcelles (1), il ne les perçoit pas (2)

structurelles

Taille des ménages

Main d'œuvre salariée

Revenu agricole

Revenu non agricole

Accès au marché

Durée d'adoption

Activité secondaire

Surface exploitée

Nombre d'individus membres de la famille travaillant dans les champs

Effectif de main d'œuvre salariée

Revenu total du coton, plus les revenus des autres cultures

Revenu provenant des autres activités des agriculteurs (variable à niveaux)

Qualité du déplacement du champ au marché : I'état de la route est bon (1), il ne l'est pas (0)

Nombre d'années depuis l'adoption

L'agriculteur a d'autres activités en dehors de l'agriculture (1), il n'en a pas (0)

Superficie totale exploitée (ha)

\section{Variables institutionnelles ou capital social}

Crédit

Sécurité foncière

Assistance

Groupement

Réseau social
L'agriculteur a accès au crédit (1), il ne l'a pas (0)

Mode d'obtention de la parcelle : succession (1), location (2), achat (3), don (4), droit de hache (5)

Assistance matérielle (1), assistance technique (2), assistance financière (3)

Appartenance à un groupement de producteurs : oui (1), non (0)

Adoption des aménagements antiérosifs à travers un réseau social : I'agriculteur a d'autres réseaux d'information agricole (1), il n'en a pas (0) 
a été de 40 ans environ. Les CE de moins de 35 ans ont été les plus nombreux ; ce sont eux qui ont aménagé leurs parcelles. Les principaux groupes ethniques rencontrés dans les villages ont été des migrants venus de l'extrême Nord et du Tchad. Les différences d'adoption entre les groupes ethniques pouvaient être importantes, allant de 29,9 p. 100 chez les Moundang à 78,8 p. 100 chez les Mafa. Une forte proportion de chrétiens, majoritaires dans les groupes ethniques ayant immigré dans la zone, a été observée. L'appartenance à un groupe religieux a semblé influencer faiblement l'adoption des aménagements : de 52,4 p. 100 (chrétien) à 60,9 p. 100 (musulman). Les personnes mariées, polygames ou monogames ont représenté 89,4 p. 100 de l'échantillon et ont été les plus nombreuses à mettre en place des aménagements : 53,5 p. 100 chez les monogames et 55,4 p. 100 chez les polygames, contre 46,5 p. 100 chez les célibataires. L'adoption des aménagements par les veufs et les divorcés a été difficilement extrapolable, car les effectifs concernés étaient faibles (4 sur les 303 enquêtés). La moitié environ des CE de l'échantillon étudié ont été conscients des problèmes d'érosion et ces derniers ont été plus nombreux que les autres à avoir recours à des aménagements. La perception des problèmes d'érosion a semblé donc avoir une influence positive sur l'adoption des aménagements. Le niveau d'instruction des CE était très bas : près des trois quarts (73 p. 100) avaient un niveau inférieur au secondaire, et parmi eux 37 p. 100 n'avaient pas été scolarisés. Les CE ayant été scolarisés ont davantage adopté les aménagements (55,0 p. 100 de ceux qui avaient le niveau du primaire et 57,3 p. 100 de ceux qui avaient au moins le niveau du secondaire) que ceux qui n'avaient pas été scolarisés (50,9 p. 100).

\section{Caractéristiques structurelles}

Les caractéristiques structurelles ont été observées à partir de la taille des ménages, de leurs revenus annuels bruts agricoles et non agricoles, de l'accès au marché, de la surface de la terre exploitée et de l'éventuelle activité secondaire des CE (tableau III). En moyenne, un ménage de l'échantillon comptait sept membres alors que la moyenne nationale était de 4,4 (8). Dans les groupes ethniques qui venaient principalement de l'Extrême Nord, région la plus peuplée du Cameroun, les ménages comptaient typiquement près de 15 individus. Dans les ménages de plus de neuf personnes le taux des aménagements $(63,2$ p. 100) a été supérieur à celui de la moyenne de l'échantillon. Un ménage de grande taille a ainsi été plus disposé à investir dans les aménagements antiérosifs, probablement du fait de la présence de main d'œuvre.

Le revenu annuel brut du coton a été inférieur à 200000 FCFA pour trois quarts des producteurs interrogés et supérieur à 500000 FCFA pour 4 p. 100 d'entre eux. Les taux d'adoption des aménagements ont été de 47,1 p. 100 pour les CE dont les revenus du coton étaient compris entre 200000 et 300000 FCFA, et de 78,9 p. 100 pour ceux avec des revenus du coton compris entre 300000 et 400000 FCFA. Ces taux ont été de 38,5 p. 100 pour les $\mathrm{CE}$ dont les revenus non agricoles étaient compris entre 200000 et 300000 FCFA, et de 92,3 p. 100 pour ceux dont les revenus non agricoles étaient supérieurs à 500000 FCFA.

Les effets des revenus du coton et non agricoles, malgré des différences de taux d'adoption parfois importantes, étaient difficiles à interpréter, car les effectifs de certaines classes étaient très faibles (respectivement 2, 3 et 4 p. 100 sur 303 CE enquêtés). La majorité des CE interrogés $(63,4$ p. 100) ont estimé que la route qui menait à leur exploitation était en bon état et donc susceptible de faciliter le transport des marchandises. Mais l'état de la route n'a pas semblé influer sur l'adoption des aménagements. Les CE qui exploitaient plus de quatre hectares ont semblé adopter davantage les aménagements que ceux qui exploitaient moins de quatre hectares.
Les CE qui n'avaient pas d'activités secondaires ont semblé adopter davantage les aménagements (56,6 p. 100) que ceux qui avaient des activités secondaires (49,5 p. 100), mais la différence entre les deux était faible.

\section{Caractéristiques institutionnelles}

Les caractéristiques institutionnelles prises en compte ont été l'accès des $\mathrm{CE}$ au crédit agricole, la tenure foncière ainsi que l'assistance technique aux fins agricoles qu'ils ont reçue (tableau IV). Les CE ayant reçu du crédit ont eu un taux de parcelles aménagées $(54,9$ p. 100) supérieur à ceux n'ayant pas reçu de crédit (48 p. 100). La tenure foncière a été appréhendée à travers les divers modes d'accès à la terre : la succession, le don, la location, l'achat et le droit de hache (le CE a défriché la parcelle). Un même $\mathrm{CE}$ pouvait obtenir deux parcelles par des modes différents. Plus de trois quarts des CE (77,5 p. 100) ayant déclaré le droit de hache comme seul mode d'obtention sur toutes leurs parcelles ont mis en place des aménagements (62 p. 100). Ils se considéraient comme propriétaires à part entière des parcelles qu'ils exploitaient. Ils pouvaient y investir sans risque de s'en voir déposséder. L'assistance agricole (aide en charrue, intrants, etc.) s'est manifestée sous trois types d'appui : (a) technique (agents Sodecoton * et ESA), (b) financier (crédit provenant de la Sodecoton, soutien d'ESA, aide des familles) ou (c) autre (séminaires de formation gratuits). Près de 80 p. 100 des producteurs consultés ont bénéficié au moins d'une de ces formes d'assistance. Cependant, cette assistance n'a pas semblé explicitement favoriser les aménagements, car les deux groupes ont montré le même pourcentage d'adoption (de l'ordre de 54 p. 100). L'adhésion à des groupements de producteurs et autres réseaux sociaux pouvait être utilisée comme un indicateur d'accès à l'information pour l'adoption des aménagements antiérosifs (respectivement 81,2 et 75,4 p. 100). Les agriculteurs ont été invités à nommer leurs trois plus proches réseaux sociaux et à préciser qui, au sein de ces réseaux, avait adopté les aménagements antiérosifs avant eux.

\section{Variables explicatives pour décrire l'adoption des aménagements et son intensité}

La variable intensité d'adoption a été censurée, c'est-à-dire non observée pour les 45,9 p. 100 des CE qui n'adoptaient pas les aménagements antiérosifs, et a été non censurée, c'est-à-dire observée pour les 54,1 p. 100 qui adoptaient les aménagements. Deux études économétriques ont été réalisées successivement sur la probabilité d'adoption ou non, puis sur l'intensité d'adoption. Ainsi, deux niveaux de choix de la part des individus de l'échantillon ont pu être distingués. Le premier, appelé équation de décision (ou de sélection), correspondait au fait que la personne décidait ou non d'adopter. Le second, appelé équation de niveau (ou de résultat), correspondait à l'intensité de cette adoption. Le tableau $\mathrm{V}$ présente les valeurs des paramètres estimés et leur significativité statistique ainsi que des indicateurs sur la robustesse des estimations économétriques des deux modèles d'adoption et d'intensité.

L'hétérogénéité signifie que tous les individus n'étaient pas identiques. Quand elle était observée dans un modèle, on pouvait prendre en compte ou expliquer les différences d'une population par les différences de valeurs prises par des facteurs explicatifs observables et généralement exogènes (environnement, facteurs culturels, etc.). Dans les modèles où elle n'était pas observée, la population était homogène. Afin de corriger l'hétérogénéité non observée qui pouvait biaiser les résultats de l'intensité d'adoption, l'équation de sélection devait inclure un ou plusieurs instruments valides.

\footnotetext{
* Société de développement du coton au Cameroun
} 

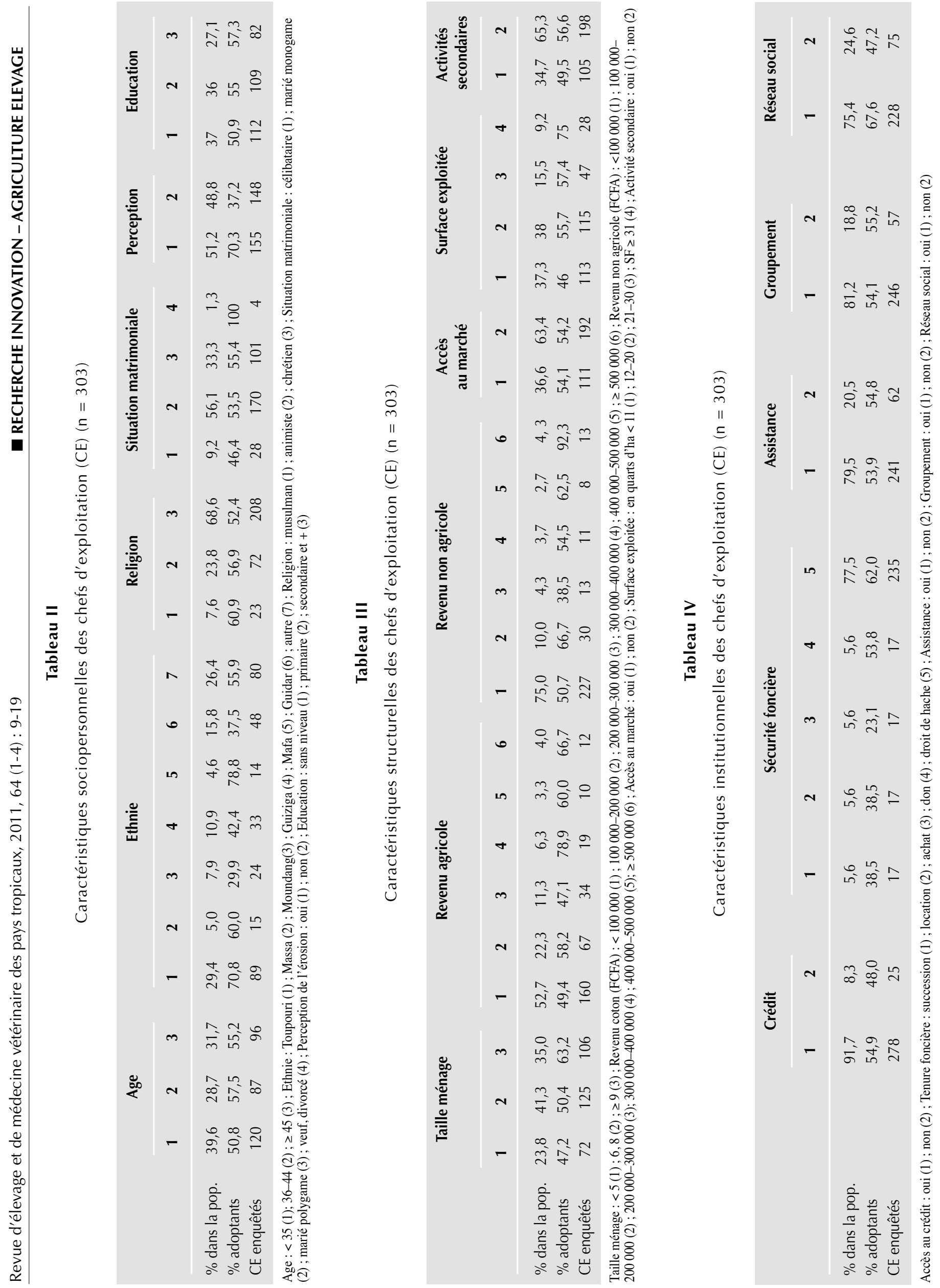
Tableau V

Estimation du modèle Tobit avec correction d'Heckman

\begin{tabular}{|c|c|c|c|c|c|}
\hline \multirow[t]{2}{*}{ Variables } & & \multicolumn{2}{|c|}{ Adoption } & \multicolumn{2}{|c|}{ Intensité d'adoption } \\
\hline & & Coefficients & Significativité & Coefficients & Significativité \\
\hline \multicolumn{6}{|l|}{ Variables sociopersonnelles } \\
\hline Age & & $-0,052$ & $0,025^{* *}$ & 0,514 & 0,935 \\
\hline $\mathrm{Age}^{2}$ & & $-0,092$ & 0,011 & $-0,055$ & 0,124 \\
\hline Education & $\begin{array}{l}\text { Sans niveau } \\
\text { Primaire } \\
\text { Secondaire }\end{array}$ & $\begin{array}{c}\mathrm{mr} \\
0,221 \\
0,331\end{array}$ & $\begin{array}{l}0,125 \\
0,114\end{array}$ & $\begin{array}{c}0,302 \\
-0,045\end{array}$ & $\begin{array}{l}0,035^{* *} \\
0,672\end{array}$ \\
\hline Appartenance Groupe/ethnie & $\begin{array}{l}\text { Toupouri } \\
\text { Massa } \\
\text { Guiziga } \\
\text { Mafa } \\
\text { Guidar } \\
\text { Autres } \\
\text { Moundang }\end{array}$ & $\begin{array}{c}3,231 \\
1,204 \\
0,432 \\
2,698 \\
0,981 \\
0,649 \\
\mathrm{mr}\end{array}$ & $\begin{array}{l}0,011^{* *} \\
0,317 \\
0,111 \\
0,023 \text { ** } \\
0,677 \\
0,761\end{array}$ & $\begin{array}{l}-0,013 \\
-1,227 \\
0,331 \\
1,039 \\
-0,295 \\
-0,166\end{array}$ & $\begin{array}{l}0,963 \\
0,322 \\
0,236 \\
0,164 \\
0,933 \\
0,695\end{array}$ \\
\hline Religion & $\begin{array}{l}\text { Musulman } \\
\text { Chrétien } \\
\text { Animiste }\end{array}$ & $\begin{array}{c}0,892 \\
0,232 \\
\mathrm{mr}\end{array}$ & $\begin{array}{c}0,001 * * * \\
0,018^{* *}\end{array}$ & $\begin{array}{l}0,041 \\
0,084\end{array}$ & $\begin{array}{l}0,819 \\
0,967\end{array}$ \\
\hline Situation matrimoniale & $\begin{array}{l}\text { Célibataire } \\
\text { Monogame } \\
\text { Polygame } \\
\text { Autre statut }\end{array}$ & $\begin{array}{c}\mathrm{mr} \\
-0,007 \\
1,704 \\
7,047\end{array}$ & $\begin{array}{l}0,982 \\
0,025 * * \\
0,124\end{array}$ & $\begin{array}{c}0,199 \\
-0,002 \\
0,362\end{array}$ & $\begin{array}{l}0,673 \\
0,957 \\
0,343\end{array}$ \\
\hline Perception Erosion & $\begin{array}{l}\text { Oui } \\
\text { Non }\end{array}$ & $\begin{array}{c}2,572 \\
\mathrm{mr}\end{array}$ & $0,000 * * *$ & $-0,197$ & 0,691 \\
\hline \multicolumn{6}{|l|}{ Variables structurelles } \\
\hline \multicolumn{2}{|l|}{$\begin{array}{l}\text { Taille ménage } \\
\text { Main d'œuvre salariée } \\
\text { Revenu agricole } \\
\text { Revenu non agricole } \\
\text { Accès au marché } \\
\text { Durée d'adoption } \\
\text { Activité secondaire } \\
\text { Surface exploitée }\end{array}$} & $\begin{array}{c}1,037 \\
0,531 \\
2,031 \\
-0,228 \\
0,123\end{array}$ & $\begin{array}{l}0,093 * \\
0,071 * \\
0,210 \\
0,334 \\
0,042 * *\end{array}$ & $\begin{array}{l}-0,002 \\
0,000 \\
0,000 \\
1,221 \\
0,051 \\
0,023 \\
1,123\end{array}$ & $\begin{array}{c}0,842 \\
0,170 \\
0,991 \\
0,567 \\
0,001 * * * \\
0,64 \\
0,254\end{array}$ \\
\hline \multicolumn{6}{|l|}{ Variables institutionnelles } \\
\hline Crédit & & $-0,897$ & 0,832 & 0,659 & 0,878 \\
\hline Sécurité foncière & $\begin{array}{l}\text { Hache } \\
\text { Location } \\
\text { Succession } \\
\text { Achat } \\
\text { Don }\end{array}$ & $\begin{array}{c}1,23 \\
0,156 \\
0,106 \\
-0,097 \\
0,286\end{array}$ & $\begin{array}{l}0,001^{* * *} \\
0,569 \\
0,327 \\
0,223 \\
0,351\end{array}$ & $\begin{array}{c}0,524 \\
-0,369 \\
-0,422 \\
-0,178 \\
0,161\end{array}$ & $\begin{array}{l}0,233 \\
0,337 \\
0,033 \text { ** } \\
0,143 \\
0,247\end{array}$ \\
\hline Assistance & $\begin{array}{l}\text { Matériel } \\
\text { Technique } \\
\text { Financière }\end{array}$ & $\begin{array}{l}0,306 \\
0,149 \\
-0,214\end{array}$ & $\begin{array}{l}0,340 \\
0,084^{*} \\
0,172\end{array}$ & $\begin{array}{l}-0,061 \\
-0,035 \\
-0,093\end{array}$ & $\begin{array}{l}0,505 \\
0,706 \\
0,755\end{array}$ \\
\hline Groupement & & 1,610 & $0,008 * * *$ & & \\
\hline Réseau social & & $-0,007$ & $0,045^{* *}$ & & \\
\hline Constante & & $-3,250$ & 0,012 & 0,342 & 0,556 \\
\hline \multicolumn{6}{|c|}{$\begin{array}{l}\text { Source : adaptation à partir de STATA } 9.0 \\
\text { Nb. d'observations }=298 ; \text { observations censurées }=141 ; \text { observations non censurées }=157 \\
\chi^{2} \text { Wald }(60)=146,20 ; \text { prob }>\chi^{2}<0,01 ; F=48,83 ; \text { prob }(F-\text { statistic })<0,01 \\
\mathrm{R}^{2}=61,37 \% ; \mathrm{R}^{2} \text { ajusté }=54,89 \% ; \text { inverse du ratio de Mills }: \text { coefficient }=0,43 ; \text { significativité }=0,89 \text {. Le ratio de Mills permet d'apprécier l'existence ou non d'un } \\
\text { biais de sélection dans l'adoption des aménagements. } \\
* \mathrm{p}<0,1 ; * * \mathrm{p}<0,05 ; * * * \mathrm{p}<0,01 ; \mathrm{r}: \text { modalité de référence }\end{array}$} \\
\hline
\end{tabular}


Deux instruments ont été identifiés : l'appartenance à un groupement de producteurs et l'adoption des aménagements antiérosifs à travers un réseau social. Pour les adoptants des aménagements antiérosifs, ces variables faisaient référence à la période antérieure à l'adoption afin d'éviter les problèmes d'endogénéité. En effet, en présence d'endogénéité, l'espérance du terme d'erreur conditionnelle à la variable explicative n'était plus nulle et les estimateurs habituels présentaient des biais.

Une autre question pertinente a été de savoir si ces instruments affectaient directement l'intensité ou étaient corrélés avec des facteurs non observés qui influençaient l'intensité. Par exemple, l'appartenance à un groupement de producteurs pouvait éventuellement conférer d'autres avantages, comme un meilleur accès aux financements et aux intrants. De même, les agriculteurs qui appartenaient à un réseau social plus innovant pouvaient également être mieux informés sur d'autres technologies bénéfiques. Dans la spécification du modèle de la présente étude, cela a été contrôlé en analysant la corrélation entre ces variables par les facteurs de production, l'accès à l'information à travers l'assistance. Les résultats ont montré, d'une part, que les instruments utilisés n'étaient pas corrélés avec l'intensité et, d'autre part, qu'ils étaient très significatifs dans l'équation de sélection. Ces instruments étaient donc valides. Des variables similaires ont été utilisées précédemment dans la recherche sur les technologies d'adoption et les interactions sociales (31).

La validité d'ensemble du modèle estimé a été analysée à l'aide de la statistique de Wald $\left(\chi^{2}\right)$ testant l'égalité à 0 de tous les coefficients du modèle. La méthode d'estimation a été celle de Heckman en deux étapes pour corriger les risques de biais de sélection. Le $\mathrm{R}^{2}$ ajusté qui mesure l'adéquation du modèle a été acceptable. Dans le cas de l'étude, le coefficient $\mathrm{R}^{2}$ ajusté a été de 55 p. 100 et la valeur $\mathrm{P}$ du $\chi^{2}$ a été inférieure à 0,01 p. 100. Le coefficient de l'inverse du ratio de Mills n'a pas été significatif (prob $=0,89$ ). Cela confirme que la méthode des moindres carrés ordinaires pouvait être utilisée pour l'estimation du modèle. Le modèle a intégré des variables qualitatives et quantitatives. Les modalités des variables qualitatives significatives s'interprétaient par rapport à la modalité de référence retenue.

Afin de tester la validité du modèle probit, le test de HosmerLemeshow a été effectué. Il permet de tester l'hypothèse nulle que l'ajustement est bon contre l'alternative que l'ajustement est mauvais. La probabilité de signification du test a été de 0,0625 . L'ajustement a donc été significatif au seuil de 5 p. 100. De plus, l'estimation du probit a été satisfaisante puisque le pseudo $\mathrm{R}^{2}$ de la régression a atteint 0,32 .

Sur l'ensemble des variables explicatives testées, neuf ont expliqué de manière significative l'adoption des aménagements antiérosifs par les CE. Elles ont été réparties selon les variables comme suit : sociopersonnelles [âge (-), perception de l'érosion (+), appartenance au groupe religieux $(+)$ et appartenance à un groupe ethnique, notamment les Toupouri et les Mafa $(+)$ ], structurelles [main d'œuvre familiale utilisée dans les exploitations $(+)$, main-d'œuvre salariée $(+)$, surface exploitée] et institutionnelle [sécurité foncière à travers le droit de hache $(+)$, assistance technique $(+)$, appartenance à un groupement de producteurs $(+)$ et réseau social (-)].

Sur l'ensemble des variables explicatives testées pour le modèle d'intensité, seules trois ont expliqué de manière significative l'intensité d'adoption des aménagements par les CE : les variables sociopersonnelles [niveau d'instruction, primaire notamment (-)], les variables structurelles [durée des aménagements dans l'exploitation, c'est-à-dire le nombre d'années depuis l'adoption des aménagements jusqu'au moment de l'enquête $(+)]$ et les variables institutionnelles [tenure foncière à travers l'obtention de la parcelle agricole par succession (-)].

\section{DISCUSSION}

\section{L'adoption des aménagements dépendait des connaissances et du profil du chef d'exploitation, mais aussi de la structure familiale}

L'influence négative de l'âge sur l'adoption dans la présente étude est conforme avec les conclusions d'une étude au Kenya (37) qui rapporte que l'âge influence négativement l'adoption de pratiques de restauration de la fertilité des sols. Ce résultat est également en accord avec des études en Ethiopie (7), aux Philippines (27), au Burkina Faso (4) et au Cameroun (15). La probabilité d'adoption plus élevée chez les jeunes agriculteurs peut s'expliquer par la tendance de ces derniers à être plus novateurs en raison de leurs horizons de planification sur le plus long terme (12). La connaissance par le CE des problèmes d'érosion sur ses parcelles a favorisé l'adoption des aménagements, ce qui rejoint les résultats d'une étude conduite au Népal (25) qui, en utilisant un modèle logit, a établi que les agriculteurs de la région centrale de ce pays, conscients de ces problèmes, sont plus aptes à investir dans des techniques de conservation des sols. En effet, les agriculteurs du Nord Cameroun ont conscience de l'état de leurs sols à partir de critères visibles (flore, faune, état de surface, texture, couleur) et économiques (productivité du travail et productivité de la terre) $(29,30,34)$.

Certains éléments de la structure des exploitations, comme la superficie cultivée, la taille de la famille et l'appartenance à un groupe ethnique, ont des effets positifs sur l'adoption des aménagements antiérosifs. Les Mafa sont originaires des monts Mandara où les aménagements des parcelles avec des cordons pierreux sont des pratiques anciennes. Les exploitations ayant le plus de main d'œuvre adoptent davantage les aménagements antiérosifs, car leur réalisation (cordons pierreux et ados) demande une forte mobilisation de la main d'œuvre.

Ainsi, les agriculteurs de plus de 35 ans et ceux qui étaient mariés, qui cultivaient des surfaces plus grandes et qui avaient plus de main d'œuvre avaient davantage de parcelles aménagées, comme rapporté par de nombreux auteurs $(2,3,12,21,45)$. La maind'œuvre salariée a aussi influencé positivement l'adoption. Les agriculteurs riches, peu nombreux, l'employaient en échange d'argent ou de nourriture. Chez les agriculteurs pauvres, elle se monnayait parfois sous forme d'arrangement entre voisins. Ces résultats corroborent ceux de Keil et coll. (24) qui montrent que l'adoption des jachères d'arbres légumineux pour l'amélioration de la fertilité des sols augmente la disponibilité du travail en Zambie. Au Kenya, Okuro et coll. (38) constatent également que l'embauche de main-d'œuvre a une relation positive avec la probabilité d'adoption de l'utilisation intégrée d'engrais organique et inorganique. Pour Oluoch-Kosura et coll. (39), la disponibilité du travail à temps plein a affecté l'adoption des pratiques de conservation des sols dans l'ouest du Kenya.

Cependant, les revenus agricoles et non agricoles des CE, en moyenne faibles dans cette région du Cameroun, n'ont pas eu d'influence sur l'adoption des aménagements antiérosifs étudiés. En effet, ces revenus n'étaient pas suffisants pour leur permettre de financer seuls des aménagements antiérosifs, c'est-à-dire essentiellement la main-d'œuvre et l'expertise des agents villageois d'aménagement ; ces coûts étaient en général pris en charge par les fonds de groupements de producteurs. L'influence positive de la tenure foncière sur l'adoption des aménagements antiérosifs par les CE rejoint les conclusions d'autres auteurs $(3,21,45)$. En effet, un CE non-propriétaire n'était pas prêt à aménager des terres qu'il n'était pas certain d'exploiter l'année suivante. Dans la zone d'étude, les services aux agriculteurs (crédits, assistance technique, formation) étaient assurés essentiellement par la Sodecoton, et par la 
Confédération nationale des producteurs de coton du Cameroun ; ils étaient destinés presque exclusivement à la production cotonnière, raison pour laquelle ils n'avaient pas d'effets sur l'adoption d'aménagements antiérosifs.

\section{L'intensité d'adoption des aménagements était fonction des connaissances, du niveau d'éducation, des compétences des $C E$ et des conditions d'accès à la terre}

D'après le modèle d'intensité d'adoption, une fois les aménagements adoptés, l'intensité d'adoption des CE dont le niveau de scolarisation était le primaire a été en moyenne 0,3 fois plus importante que celle des CE sans niveau d'instruction.

En revanche, la durée des aménagements chez un CE a influencé son intensité d'adoption. Ainsi, une année supplémentaire d'aménagement a augmenté l'intensité de 5 p. 100. D'autres études (17, 46) ont montré l'intensité d'adoption avec un modèle Tobit. Les CE qui ont observé au cours du temps les effets positifs des aménagements ont eu une forte tendance à accroître la surface aménagée par rapport à la surface dont ils disposaient.

L'accès à la propriété a également influencé l'intensité d'adoption chez les CE. Le modèle de régression a montré que l'intensité d'adoption des CE ayant reçu leurs parcelles par succession a été de 42 p. 100 inférieure à celle de leurs homologues n'ayant pas reçu leurs parcelles par succession. Ceci pouvait être dû à des résistances culturelles. En réalité, dans la zone d'étude, les héritiers des terres n'avaient pas toujours la capacité ni le désir de valoriser leurs parcelles, contrairement au $\mathrm{CE}$ ayant acheté et/ou défriché (droit de hache) leurs parcelles. Ces derniers connaissaient la valeur de leur terre et y investissaient plus facilement.

\section{CONCLUSION}

Dans le bassin versant du lac Lagdo au Nord Cameroun, les programmes d'aménagements antiérosifs ont été mis en œuvre par le biais de groupements de producteurs, car les exploitants agricoles, cultivant de petites superficies qui produisaient des revenus faibles, n'avaient pas les moyens de réaliser seuls ces aménagements. Sur 303 ménages interrogés, 54 p. 100 ont adopté les aménagements antiérosifs, indiquant une bonne adoption. Si cette tendance se maintenait, une augmentation souhaitable de cette adoption pourrait apparaître dans la zone soudano-sahélienne du Cameroun. Les facteurs qui ont influencé de manière significative la décision d'adopter ou non des aménagements antiérosifs ont été, positivement, la main d'œuvre familiale et salariale, la perception des problèmes d'érosion, l'appartenance à un groupe religieux et/ ou ethnique et la sécurité foncière, et, négativement, l'âge du chef d'exploitation. L'intensité de cette adoption a été influencée positivement par le niveau d'instruction, la durée des aménagements et la sécurité foncière.

Les programmes d'aménagements antiérosifs doivent prendre en compte ces facteurs dans leurs méthodes d'intervention pour mieux définir les groupes cibles, mais aussi dans les évaluations d'impact socioéconomique sur les conditions de vie des agriculteurs, afin de mieux orienter les actions futures de lutte contre l'érosion. Ces actions futures doivent aussi prendre en compte le rôle des groupements de producteurs dans l'adoption des aménagements antiérosifs par les agriculteurs, les GP étant à l'interface entre les agriculteurs et les programmes de promotion et de diffusion des aménagements. Elles doivent aussi mieux intégrer les interactions entre les types d'aménagements antiérosifs étudiés ici et les techniques d'agroforesterie et d'agriculture de conservation (système sous couverture végétale, par exemple) développées au Nord Cameroun.

\section{Remerciements}

Les auteurs remercient la coordination du projet Eau-Sol-Arbre de Garoua, pour son soutien financier et l'apport de matériels, ainsi que la Mission d'études pour l'aménagement et le développement de la province du Nord sans laquelle ce travail n'aurait pu être mené à terme.

\section{BIBLIOGRAPHIE}

1. ABOU ABBA A., HOFS J.L., MERGEAI G., 2006. Relever les défis environnementaux pour les filières cotonnières $d^{\prime}$ Afrique de l'Ouest et du Centre. Biotechnol. Agron. Soc. Environ., 10 : 351-359.

2. ADESINA A.A., MBILA D., NKAMLEU G.B., ENDAMANA D., 2000. Economic analysis of the determinants of adoption of alley farming by farmers in the forest zone of southwest Cameroon. Agric. Eco. Environ., 80: $255-265$.

3. ASRAT P., BALAY K., HAMITO D., 2004. Determinants of farmer's willingness to pay for soil conservation practices in the south eastern Highlands of Ethiopia. Land Degr. Dev., 15: 423-438.

4. AYUK E.T., 1997. Adoption of agroforestry technology: the case of live hedges in the Central Plateau of Burkina Faso. Agric. Syst., 54: 189-206.

5. BABOULE Z.B., ROOSE E., BEP A., ZIEM B., SEMON K., WAECHTER F., 1993. Effets des techniques culturales sur le ruissellement, l'érosion et la production de coton et de maïs sur un sol ferrugineux tropical sableux. Recherche de systèmes de culture intensifs et durable en région soudanienne au Nord Cameroun. Cah. Orstom, Sér. Pédol., 27 : 309-325.

6. BAIDU-FORSON J., 1999. Factors influencing adoption of landenhancing technology in the Sahel: lessons from a case study in Niger. Agric. Econ,. 20: 231-239.
7. BEKELE S., HOLDEN S., 1998. Resource degradation and adoption of land conservation technologies in the Ethiopian Highlands. A case study in Andit Tid, North Shewa. Agric. Econ., 18: 233-247.

8. BRETT C., 2004. Farm level adoption decisions of soil water management technologies in semi-arid Eastern Kenya. In: 48th Annu. Conf. Australian Agricultural and Resource Economics Society, Melbourne, Australian, 11-13 Feb. 2004.

9. DERPSCH R., 2005. The extent of conservation agriculture adoption worldwide: implications and impact. In: 3rd World Congr. Conserv. Agric., World Agroforestry Center, Nairobi, Kenya.

10. DOSS C., 2006. Analyzing technology adoption using microstudies: Limitations, challenges, and opportunities for improvement. Agric. Econ., 34: 207-219.

11. ECAM III, 2008. Troisième enquête camerounaise auprès des ménages : tendances, profil et déterminants de la pauvreté au Cameroun entre 2001-2007. Yaoundé, Cameroun, Institut national de la statistique.

12. ERVIN C.A., ERVIN D.E., 1982. Factors affecting the use of soi conservation practices: hypothesis, evidence and policy implication. Land ECo., 58: 277-292.

13. FEDER G., JUST R., ZILBERMAN D., 1985. Adoption of agricultural innovations in developing countries: a survey. Econ. Dev. cult. Change, 33: 255-298. 
14. GREENE W., 2008. Econometric analysis. Upper Saddle River, NJ, USA, Prentice Hall.

15. GOCKOWSKI J., NDOUMBE M., 2004. The adoption of intensive horticulture in Southern Cameroon. Agric. Econ., 30: 195-202.

16. GOWING J.W., PALMER M., 2008. Sustainable agricultura development in sub-Saharan Africa: the case for paradigm shift in land husband. Soil Use Manage., 24: 92-99.

17. HAGGBLADE S., TEMBO G., 2003. Early evidence on CF in Zambia In: Int. Work. Reconciling Rural Poverty and Resource Conservation: Identifying Relationships and Remedies, Cornell University, Ithaca, New York.

18. HECKMAN J., 1979. Sample selection bias as a specification error Econometrica, 47: 153-161.

19. HOBBS P.R., SAYRE K., GUPTA R., 2007. The role of conservation agriculture in sustainable agriculture. Phil. Trans. R. Soc. B., 363: 543555.

20. HOVE L., TWOMLOW S., 2007. Is conservation agriculture an option for vulnerable households in Southern Africa? In: Work. Conservation Agriculture for Sustainable Land Management to Improve the Livelihood of People in Dry Areas, FAO, Damascus, Syria, 7-9 May 2007

21. ILLUKPITIYA P., GOPALAKRISHNAN C., 2004. Decision making in soil conservation: application of a behavioral model to potato farmers in Sri Lanka. Land Use Policy, 21: 321-331.

22. IRAD, 2001. Etude diagnostique des aménagements hydroagricoles de Lagdo. Garoua, Cameroun, IRAD, 77 p.

23. KABUNGA N.S., DUBOIS T., QAIM M., 2012. Heterogeneous information exposure and technology adoption: The case of tissue culture bananas in Kenya. J. Agric. Econ., 63: 444-464.

24. KEIL A., ZELLER M., FRANZEL S., 2005. Improved tree fallows in smallholder maize production in Zambia: Do initial testers adopt the technology? Agroforest. Syst., 64: 225-236.

25. KRISHNA R. TIWARI BISHAL $K$, SITAULA I.L NYBORG GIRIDHARI P., PAUDEL S., 2008. Determinants of farmers' adoption of improved soil conservation technology in a middle mountain watershed of Central Nepal. Environ. Manage., 42: 833-846.

26. LAL R., 2007. Constraints to adopting no-till farming in developing countries. Soil Tillage Res., 94: 1-3.

27. LAPAR M.L.A., PANDEY S., 1999. Adoption of soil conservation: The case of the Philippines uplands. Agric. Econ., 21: 241-256.

28. LICHTENBERG E., 2001. Adoption of soil conservation practices: a revealed preference approach. College Park, MD, USA, University of Maryland.

29. M'BIANDOUN M., GUIBERT H., OLINA J.P., 2006. Caractérisation de la fertilité du sol en fonction des mauvaises herbes présentes. Tropicultura, 24 : 247-252.

30. M'BIANDOUN M., OLINA J.P., 2007. Savoir paysan et fertilité des terres au Nord-Cameroun. Cah. Agric., 16 : 185-197.

31. MATUSCHKE I., QAIM M., 2009. The impact of social networks on hybrid seed adoption in India. Agric. Econ., 40: 493-505.

32. MAZVIMAVI K., TWOMLOW S., 2009. Socioeconomic and institutional factors influencing adoption of conservation by farming by vulnerable households in Zimbabwe. Agric. Syst., 101: 20-29.

33. MAZVIMAVI K., TWOMLOW S, 2008. Conservation farming for agricultural relief and development in Zimbabwe. In: Goddard T. Zoebisch M.A., Gan Y.T., Ellis W., Watson A., Sombatpanit S. Eds, Notill farming systems. Special publication No 3. Bangkok, Thailand, World Association of Soil and Water Conservation, p. 544

34. MBETID-BESSANE E., HAVARD M., DJONDANG K., 2006. Evolution des pratiques de gestion dans les exploitations agricoles familiales des savanes cotonnières d'Afrique centrale. Cah. Agric., 15 : 555-561.
35. MCDONALD J.F., MOFFIT R.A., 1980. The uses of Tobit analysis. Rev. Econ. Stat. 62: 318-321.

36. MEADEN, 2005. Evaluation de la sédimentation du lac de Lagdo et détermination de la fréquence de son curage. Yaoundé, Cameroun, Meaden.

37. ODERA M.M., KIMANI S.K., MUSEMBI F., 2000. Factors influencing adoption of integrated use of manure and inorganic fertilizer in central highlands of Kenya. In: Proc. 7th Biennial Scientific Conf., Collaborative and Participatory Research for Sustainably Improved Livelihoods. Nairobi, Kenya, Kenya Agricultural Research Institute, p. 58-64.

38. OKURO J.O., MURIITHI F.M., MWANGI W., VERJUIKL H., GETHI M., GROOTE H., 2002. Adoption of maize seed and fertilizer technologies in Embu district. Nairobi, Kenya, Kenya Agricultural Research Institute.

39. OLUOCH-KOSURA W.A., MARENYA P.P., NZUMA M.J., 2001. Soil fertility management in maize-based production systems in Kenya. Current options and future strategies. In: 7th Eastern and Southern Africa Regional Maize Conf., Nairobi, Kenya, 11-15 Feb., p. 350-355.

40. PUHANI P., 2000. The Heckman correction for sample selection and its critique. J. Econ. Surveys, 14: 53-68.

41. RUSIKE J., TWOMLOW S.J., FREEMAN H.A., HEINRICH G.M., 2006. Does farmer participatory research matter for improved soil fertility technology development and dissemination in Southern Africa? Int. J. Agric. Sustain., 4: 176-192.

42. ROCKSTROM J., HATIBU N., OWEIS T.Y., WANI S., BARRON I. BRUGGEMAN A., FARAHANI J., KARLBERG L., QIANG Z., 2007. Managing water in rainfed agriculture. In: CAWMA 2007, Water for food, water for life: A comprehensive assessment of water management in agriculture. London, UK, Earthscan.

43. SALL S., NORMAN D., FEATHERSTONE A.M., 2000. Quantitative assessment of improved rice variety adoption: the farmer's perspective. Agric. Syst., 66: 129-144.

44. SCHERR S., 2000. A downward spiral? Research evidence on the relationship between poverty and natural resource degradation. Food Policy, 25: 479-498.

45. SIDIBE A., 2005. Farm-level adoption of soil and water conservation techniques in northern Burkina Faso. Agric. Water Manage., 71: 211-224.

46. TEKLEWOLD H., DADI L., YAMI A., DANA N., 2006. Determinants of adoption of poultry technology: a double-hurdle approach. Livest. Res. Rural Dev., 18.

47. THEVOZ C., 2000. Le zaï ou les limites d'une stratégie de mise en culture des sols dégradés au Burkina Faso dans la sécurité alimentaire en question : dilemmes, constats et controverses. Paris, France, Karthala, p. 217-229.

48. TOBIN J., 1956. Estimation of relationship for limited dependent variables. Econometrica, 26: 24-36.

49. TWOMLOW S.J., STEYN J.T., DU PREEZ C.C., 2006. Dryland farming in southern Africa. In: Dryland agriculture, 2nd Edn. Madison, WI, American Society of Agronomy, p. 769-836. (Agronomy Monograph No 23)

50. TWOMLOW S., UROLOV J.C., JENRICH M., OLDRIEVE B., 2008. Lessons from the field. Zimbabwe's Conservation Agriculture Task Force. J. SAT agric. Res., 6.

51. WINTERS P., CRISSMAN C., Espinosa P., 2004. Inducing the adoption of conservation technologies: Lessons from the Ecuadorian Andes. Environ. Dev. Econ., 9: 695-719.

Mis en ligne en juin 2013 


\section{Summary}

Ngondjeb Y., Nje P., Havard M. Determinants of the adoption of control techniques against water erosion in the cotton zone of Cameroon

In the cotton zone of Cameroon, the increasing pressure on land has led to an increase in agricultural areas and increased soil erosion caused by rainfall intensity on poorly protected soils. For the last two decades, anti-erosion plans have been disseminated, but few studies have analyzed the factors promoting their adoption. The objective of this study was to identify the factors that influenced the adoption and the adoption intensity of anti-erosion plans by farmers. A survey from the Water-Soil-Tree project involving 303 farmers was carried out in the cotton zone between 2008 and 2009. Survey data were analyzed with Tobit Model II. Results showed that among the variables studied, those that impacted on farmers' decision to adopt anti-erosion plans were (i) their age, (ii) their perception of erosion problems, (iii) their belonging to a group (religious, ethnic), (iv) labor availability, and (v) land tenure. Factors that explained the adoption intensity by farmers were the number of years since adopting the plans and their access mode to land. The educational level did not affect the adoption nor the adoption intensity. In future actions, erosion control programs should integrate these results in their intervention methods, but also in socio-economic impact assessments.

Keywords: Innovation adoption - Watershed management Econometric model - Erosion control - Cameroon.

\section{Resumen}

Ngondjeb Y., Nje P., Havard M. Determinantes de la adopción de técnicas para la lucha contra la erosión hídrica en zona algodonera de Camerún

En la zona algodonera de Camerún, la presión territorial creciente a conducido a un aumento de las superficies cultivadas y acentuado la erosión de los suelos causada por la intensidad de las Iluvias sobre los suelos mal protegidos. Desde hace dos décadas, los equipamientos de control de la erosión se han divulgado, pero pocos estudios han analizado los factores favorables a su adopción. El objetivo del presente estudio es el de identificar los factores que infuencian la adopción y la intensidad de la adopción de los equipamentos de control de la erosión por parte de los agricultores. Entre 2008 y 2009, se efectuó una encuesta en el marco del proyecto Agua-SueloArbol (ESA), entre los agricultores de la zona algodonera. Los datos de esta encuesta fueron analizados gracias a un modelo Tobit II. Los resultados mostraron que, entre las variables estudiadas, las que influenciaron la decisión de la adopción de los equipamentos de control de la erosión por parte de los agricultores fueron (a) la edad de los agricultores, (b) la percepción de los problemas de erosión por parte del agricultor, (c) su afiliación a un grupo (religión, etnicidad), (d) la disponibilidad de la mano de obra y (e) la tenencia territorial. Los factores explicando la intensidad de esta adopción por parte de los agricultores fueron el nombre de años pasados desde la adopción de los equipamentos y la forma de acceso al territorio. El nivel de instrucción no influyó sobre la adopción, ni sobre la intensidad de esta adopción. Con el fin de orientar mejor las acciones futuras, los programas de lucha contra la erosión deben tomar en cuenta estos resultados en sus métodos de intervención, así como también en las evaluaciones de los impactos socio económicos.

Palabras clave: Adopción de innovaciones - Ordenación de cuencas - Modelo econométrico - Control de la erosión Camerún. 
